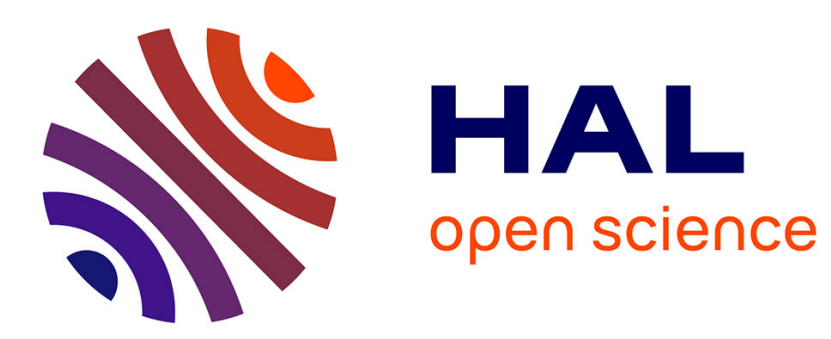

\title{
Faut-il (vraiment) augmenter les frais d'inscription à l'université ?
}

David Flacher, Hugo Harari-Kermadec, Léonard Moulin

\section{To cite this version:}

David Flacher, Hugo Harari-Kermadec, Léonard Moulin. Faut-il (vraiment) augmenter les frais d'inscription à l'université ?. Revue Française d'Economie, 2012, 27 (3), pp.145-183. 10.3917/rfe.123.0145 . hal-00749625v2

\section{HAL Id: hal-00749625 \\ https://hal.science/hal-00749625v2}

Submitted on 14 Jul 2016

HAL is a multi-disciplinary open access archive for the deposit and dissemination of scientific research documents, whether they are published or not. The documents may come from teaching and research institutions in France or abroad, or from public or private research centers.
L'archive ouverte pluridisciplinaire HAL, est destinée au dépôt et à la diffusion de documents scientifiques de niveau recherche, publiés ou non, émanant des établissements d'enseignement et de recherche français ou étrangers, des laboratoires publics ou privés. 


\section{FAUT-IL (VRAIMENT) AUGMENTER LES FRAIS D'INSCRIPTION À L'UNIVERSITÉ ?}

David Flacher, Hugo Harari-Kermadec, Léonard Moulin

Revue française d'économie | «Revue française d'économie »

2012/3 Volume XXVII | pages 145 à 183

ISSN 0769-0479

Article disponible en ligne à l'adresse :

http://www.cairn.info/revue-francaise-d-economie-2012-3-page-145.htm

\section{Pour citer cet article :}

David Flacher et al., « Faut-il (vraiment) augmenter les frais d'inscription à l'université ? », Revue française d'économie 2012/3 (Volume XXVII), p. 145-183. DOI $10.3917 / \mathrm{rfe} .123 .0145$

Distribution électronique Cairn.info pour Revue française d'économie.

(C) Revue française d'économie. Tous droits réservés pour tous pays.

La reproduction ou représentation de cet article, notamment par photocopie, n'est autorisée que dans les limites des conditions générales d'utilisation du site ou, le cas échéant, des conditions générales de la licence souscrite par votre établissement. Toute autre reproduction ou représentation, en tout ou partie, sous quelque forme et de quelque manière que ce soit, est interdite sauf accord préalable et écrit de l'éditeur, en dehors des cas prévus par la législation en vigueur en France. Il est précisé que son stockage dans une base de données est également interdit. 


\section{David}

\section{FLACHER}

\section{Hugo}

HARARI-KERMADEC

Léonard

MOULIN

\section{Faut-il (vraiment) augmenter les frais d'inscription à l'université?}

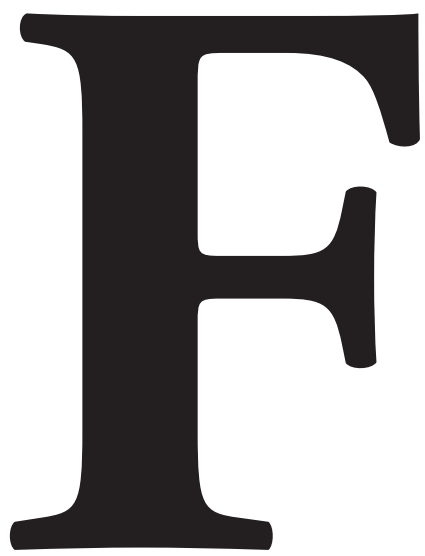

aut-il augmenter les frais d'inscription à l'université ? Cette question renvoie à des problématiques cruciales sur l'allocation des ressources dans une économie fondée sur la connaissance et les compétences. Elle intéresse plus généralement les sciences humaines et sociales, dans la 
mesure où l'introduction ou le relèvement des frais d'inscription est de nature à transformer en profondeur les rapports sociaux et notamment le rapport des étudiants à l'éducation. Cette question s'insère enfin dans un débat public qui ne cesse de gagner en actualité. D'après un rapport de l'OCDE [2009], la diminution constatée de la part du financement public des établissements d'enseignement supérieur entre 1995 et 2006 " s'explique essentiellement par une tendance qui s'observe dans des pays non européens, à savoir des frais de scolarité plus élevés et une grande participation des entreprises au financement des établissements d'enseignement tertiaire " (OCDE [2009], p. 236). Cette tendance rattrape désormais l'Europe : le Royaume-Uni a adopté, en 2010, une réforme des frais d'inscription, rehaussant leur plafond de 3300 livres à 9000 livres et développant les capacités d'emprunt des étudiants, alors même que les dépenses publiques pour l'enseignement supérieur se réduisent fortement. En France, bien que la question soit très sensible, les expériences menées, en la matière, à l'Université Paris-Dauphine et à Sciences Po Paris soulignent l'intérêt pour ces politiques, au-delà des clivages partisans ${ }^{1}$.

Dans un article publié dans la Revue française d'économie, Gary-Bobo et Trannoy [2005] ont développé une argumentation en faveur de l'augmentation des frais d'inscription. Cette argumentation se concentrait sur l'efficience des modes de sélection à l'entrée de l'université : sous réserve d'un accès parfait au crédit, les frais d'inscription pourraient conduire les étudiants à s'auto-sélectionner en fonction de leur talent. D'autres travaux soulignent l'importance des rendements privés de l'éducation et la légitimité qui résulterait, en termes d'équité, de l'instauration d'une contribution des étudiants (Gregoir [2008] et Courtioux [2009, 2010]). Dans la même veine, un numéro spécial de la Revue d'économie politique, dirigé par d'Autume et al. [2006], fait référence aux besoins financiers de l'université française et propose des arrangements institutionnels permettant de faire contribuer davantage les étudiants aux coûts de leurs études.

Cependant, ces résultats apparaissent discutables dès lors que l'on s'intéresse au détail des argumentations et que l'on 
mobilise d'autres disciplines au sein des sciences humaines et sociales. C'est ainsi que Flacher et Harari-Kermadec [2013] ont montré, en tenant compte de l'hétérogénéité des comportements des étudiants selon les classes sociales, que les résultats de Gary-Bobo et Trannoy [2005, 2008], favorables aux frais d'inscription, devaient être relativisés. Les travaux de Flacher et Harari-Kermadec [2013] apparaissent néanmoins limités dans la mesure où ils ne s'intéressent qu'à l'efficience supposée résulter des effets incitatifs (en matière de sélection des étudiants à l'entrée de l'université), et dans la mesure où leur formalisation est particulièrement restrictive. Or, comme ils le soulignent, il convient, avant de dégager une position normative sur les frais d'inscription, de considérer plus avant i) l'effet redistributif des frais d'inscription, qui peut avoir des conséquences sur l'équité du système universitaire, ii) l'effet incitatif qui, en contribuant à sélectionner, à orienter ou à motiver les acteurs du système éducatif, peut avoir un impact sur l'efficience du système et enfin, iii) l'effet contributif, qui pourrait permettre aux universités de disposer de moyens financiers supplémentaires à travers les contributions des étudiants.

Dans cet article, nous proposons d'envisager ces trois dimensions en proposant une revue critique de la littérature reposant à la fois sur des travaux publiés dans le champ de l'économie mais également sur certains travaux développés dans d'autres disciplines. Ce travail présente ainsi l'originalité de considérer le sujet dans sa globalité, de mobiliser différents apports disciplinaires pour enrichir la pensée économique, et surtout de poser un regard critique sur les politiques de tarification des études supérieures.

Nous avons choisi la terminologie "frais d'inscription " pour désigner les différentes contributions qui peuvent être demandées à l'étudiant par l'institution universitaire. Ce terme permet de recouvrir ainsi les "droits de scolarité », au sens d'impôt ou taxe, actuellement prélevés par les universités françaises, d'une part, et les "frais ", dont le sens est lié au "coût " du système éducatif. Nous préférons enfin la notion de "frais d'inscription " à celle de " frais de scolarité " dans la mesure où le 
coût réel de la "scolarité » dépasse largement les coûts de fonctionnement de l'université (il inclut notamment les dépenses de subsistance et le matériel nécessaire au cursus de l'étudiant et les coûts d'opportunité des études).

La première section propose une analyse critique des motivations théoriques à une éducation "par capitalisation ", c'est-à-dire un régime d'éducation dans lequel les étudiants (ou leurs familles) participent au financement de leurs études au travers de frais d'inscription reflétant une part significative des coûts. Nous y discutons de l'équité d'un telle politique, de l'efficience d'un système qui ferait jouer aux frais d'inscription le rôle de prix ainsi que des opportunités de financement que représentent les frais d'inscription pour le système universitaire. La deuxième section traite des atouts et limites des mécanismes d'accompagnement. La dernière section résume les principales conclusions et propose des perspectives de recherche.

\section{Analyse critique des motivations éco- nomiques pour une éducation " par capitalisation "}

Dans une logique de "capitalisation ", l'investissement éducatif permet l'accumulation de " capital humain " auquel est associé un rendement privé (lié, dans l'essentiel de la littérature économique, à un accroissement du niveau de salaire et au coût des études). La littérature sur les frais d'inscription reste pourtant relativement limitée. Elle met en évidence trois catégories essentielles d'arguments visant généralement à promouvoir une éducation fondée sur la capitalisation : i) l'argument de la justice sociale est le plus souvent avancé car les classes sociales favorisées sont sur-représentées dans l'enseignement supérieur. Dans ce contexte, la faiblesse des frais d'inscription apparaît anti- 
redistributive, et ce d'autant plus que les rendements privés de l'éducation sont élevés ; ii) une promotion de l'efficience, aussi bien allocative (en décourageant les étudiants de s'engager dans des études qui ne leur correspondent pas) que productive (en incitant les étudiants comme les enseignants et les universités à l'effort), est également mise en avant ; iii) enfin, un troisième argument repose sur le nécessaire accroissement des budgets de l'enseignement supérieur, notamment dans certains pays comme la France. L'analyse critique de ces arguments est proposée dans les sous-sections suivantes.

\section{Le système d'enseignement supérieur ne serait pas sociale- ment juste : équité contributive et rendements privés}

\section{Qu'est-ce qu'un enseignement supérieur équitable?}

Plusieurs approches de l'équité sont possibles. Dans un système fondé sur la responsabilité, il est considéré comme "juste " que chaque individu exerce librement ses responsabilités et en assume entièrement les conséquences. Un tel système repose sur ce que Fleurbaey [1995] appelle le " principe de récompense naturelle ": dans cette perspective libérale, chaque individu bénéficie de son " talent ", de ses efforts et de ses choix libres. Du point vue de l'" égalité des chances ", il s'agit de mettre en œuvre un "principe de compensation " contrebalançant, par des ressources externes, les écarts entre situations individuelles (ou " circonstances ") dont les individus ne sont pas responsables (Fleurbaey [1995]), que celles-ci soient d'ordre socio-économique ou innées. Ces deux principes ne sont cependant pas nécessairement compatibles.

De surcroît, pour Rawls [1971], l'égalité des chances ne suffit pas à définir un système équitable. Il faut aussi, pour que les inégalités économiques et sociales soient acceptables, qu'elles contribuent au bénéfice de la société et notamment des plus défavorisés. Une interprétation possible de la justice sociale selon Rawls peut donc reposer à la fois sur le principe de 
compensation (l'accès à l'éducation constituant, selon nous, un "bien premier ", au sens de Rawls) et sur celui d'équité contributive. Il entre alors en conflit avec le principe de récompense naturelle.

Enfin, si nous suivions Sen [2010], il conviendrait de dépasser les libertés négatives (les droits formels, i.e. l'absence d'entraves) et la problématique des ressources financières (qui fondent le principe de compensation) pour s'intéresser aux libertés positives (ou capabilités), c'est-à-dire à ce que chaque individu est capable d'accomplir étant donné les ressources dont il dispose et le milieu dont il est issu. Or, comme Sen le montre, les coercitions socio-culturelles peuvent conduire les personnes à ne pas même envisager ou s'autoriser l'expression de certains désirs, aspirations ou projets, remettant également en cause le principe de récompense naturelle.

Dans la suite de ce travail, nous considèrerons qu'un système d'enseignement supérieur est équitable s'il vérifie à la fois les principes $: i$ ) de compensation (égalité des chances en termes d'accès à un minimum de ressources), ii) d'équité contributive de tous les bénéficiaires de l'enseignement supérieur (définie comme un effort identique de tous les bénéficiaires - étudiants et citoyens profitant des externalités - et donc par progressivité des contributions) et iii) si des libertés positives permettent effectivement aux individus des différents milieux sociaux d'aspirer aux mêmes diplômes (égalité des chances effective).

Rendements privés, rendements sociaux et partage des coûts de l'enseignement supérieur

L'enseignement supérieur se caractérise par d'importants rendements privés à la fois monétaires et non monétaires. En termes monétaires, des travaux soulignent que les gains salariaux (avant impôt) liés à une année d'étude supplémentaire seraient compris entre $4 \%$ et $12 \%$ dans les pays développés (Card [1999], Harmon et al. [2003], Hendricks [2004], Gregoir [2008]) et que, selon les formations, les taux internes de rendement individuel des études iraient de 4,5\% à $13 \%$ (Courtioux [2009]) 
- voir aussi Boarini et Strauss [2007] et Strauss et de La Maisonneuve [2009]). Pour Bourdieu [1974], l'investissement éducatif traduirait aussi la volonté de maximiser un rendement "symbolique » qui dérive de la satisfaction intellectuelle ou du statut social que l'éducation permet d'obtenir.

Parallèlement à ces rendements privés, l'enseignement supérieur présente d'importants rendements sociaux (Grossman [2006]) ayant un impact positif sur la criminalité (Garces et al. [2002]), la santé (Grossman et Kaestner [1997]), la croissance économique (Aghion et Howitt [2009]), le dynamisme démocratique (Haveman et Wolfe [1984]) et même, indirectement, sur les recettes fiscales (Barr [1993]) ou sur l'emploi non qualifié (Johnson [1984]). La participation de l'ensemble de la collectivité (y compris des moins favorisés) au financement de l'éducation au-delà de la scolarité obligatoire apparaît donc justifiée.

A côté de l'enjeu politique que constitue un large accès à l'enseignement supérieur, l'enjeu économique réside donc dans la correction d'un possible sous-investissement privé dans l'éducation prenant en compte les " rendements sociaux ». Le taux de subvention s'interprète alors comme une mesure correctrice à la Pigou, destinée à internaliser les externalités de l'éducation (Gregoir [2008] et Barr [1993]) qui restent néanmoins tout aussi difficilement quantifiables que la rentabilité financière des études sur quarante années d'activité (Vinokur [2009, p. 444]).

\section{L'enseignement supérieur français est-il inéquitable?}

En France, comme dans d'autres pays, le système d'enseignement supérieur revêt un caractère inéquitable : il bénéficie davantage aux classes sociales les plus favorisées. Cette iniquité résulte, a minima, d'une absence de compensation des inégalités sociales et d'une relative absence de libertés positives dans certains milieux. Elle est d'autant plus criante que l'on considère les capacités individuelles uniformément réparties entre les milieux sociaux, que le système fiscal est faiblement progressif et que les aides (bourses et allègements fiscaux) restent limitées et peu redistributives ${ }^{2}$. 
Le caractère anti-redistributif apparaît encore plus marqué si l'on considère plus finement le niveau des diplômes obtenus par les enfants des différentes catégories sociales. En effet, les résultats de la démocratisation sont ambigus : si la part des classes populaires et intermédiaires diplômées de l'enseignement supérieur augmente, la sur-représentation des étudiants issus d'une origine sociale supérieure (au sens de l'Insee) croît avec le niveau du diplôme et la dépense publique : selon Gregoir [2008] et Courtioux [2009], $72 \%$ des enfants d'origine sociale supérieure sont diplômés de l'enseignement supérieur, en ayant bénéficié d'une dépense moyenne de 31000 euros, alors que seulement $25 \%$ des enfants d'origine populaire sont diplômés de l'enseignement supérieur, pour une dépense moyenne par diplômé de 19000 euros $^{3}$. De même, le " coût annuel de formation d'un élève des " très grandes écoles" apparait 14 fois supérieur à celui d'un élève qui suit un premier cycle en droit ou sciences économiques ${ }^{4}$. Enfin, les inégalités se trouvent renforcées par les aides fiscales dont profitent les ménages aisés, qui représentent $60 \%$ du montant total des bourses attribuées aux ménages modestes (Gregoir [2008]).

En l'absence de compensation suffisante des inégalités par le système social, le coût élevé d'entretien conduit certains étudiants à abandonner leurs études, à modifier l'orientation qui aurait été la leur en l'absence de difficultés financières, ou à travailler pour financer leur scolarisation (au risque d'accroître l'échec ${ }^{5}$ ). La "gratuité " de l'université et la modestie des bourses apparaissent inéquitables : elles ne permettent ni d'offrir un accès aux ressources nécessaires pour entreprendre et mener à bien des études, ni de garantir l'équité contributive.

\section{Les frais d'inscription (non mutualisés) et les aides financières constituent-il une opportunité pour davantage d'équité ?}

Rétablir une forme d'équité doit donc s'envisager selon trois dimensions et selon différentes modalités.

- Rendre les contributions progressives. Pour ce faire, le partage des coûts entre l'étudiant ou l'ancien étudiant (en tant que bénéficiaire direct des rendements éducatifs) et la collectivité (en 
tant que bénéficiaire des externalités) peut s'envisager dans une temporalité synchrone (en faisant porter la charge sur l'étudiant pendant ses études, notamment avec des frais d'inscription) ou asynchrone (à travers une taxe ou une cotisation qui pourrait dépendre de la participation passée du contribuable à l'enseignement supérieur).

- Le système, en vertu du principe de compensation, peut développer et mieux doter les mécanismes d'aide financière aux étudiants.

- Un important travail doit également résider dans la compensation des difficultés non financières qui expliquent le niveau et la structure sociale de participation à l'enseignement supérieur : l'éducation des enfants dès le plus jeune âge et les conditions de réussite doivent être pensées tout au long de la scolarité, en octroyant les moyens nécessaires pour compenser des inégalités culturelles et sociales. Si cette dimension est essentielle, elle dépasse très largement le cadre de notre article, nous nous concentrerons donc essentiellement sur les deux premières.

Pour rétablir une certaine progressivité et dégager des ressources pour aider les élèves talentueux issus de milieux modestes ou discriminés, des économistes concluent à la nécessité d'instaurer une contribution individuelle au coût de la scolarité (Johnstone [2004], Krueger et Lindahl [2001], Temple [2001], Spence [1973] et Stiglitz [1975]). Ces conclusions sont cependant à nuancer dans la mesure où les motifs d'un "partage des coûts " ne peuvent s'envisager qu'en tenant compte simultanément des gains individuels et sociaux, monétaires et non monétaires liés à l'enseignement supérieur. Il faut en particulier considérer non seulement l'ensemble des coûts supportés par les étudiants et leurs familles, le fait que les familles les plus aisées participent plus, par leur impôt, au financement des dépenses publiques d'éducation mais également l'effort consenti par chacun au regard de ses facultés contributives.

La pratique consistant à réunir et utiliser localement ces frais d'inscription est intrinsèquement (ou pratiquement) porteuse d'iniquité. Vinokur [2009] explique que « les étudiants étant simultanément financeurs de l'établissement et matière première dont la qualité est déterminante pour son prestige, on constate que 
l'aide " au mérite » tend à prédominer sur l'aide " aux besoins » favorisant ainsi les étudiants des milieux les plus aisés où la proportion de candidats brillants est la plus élevée ${ }^{6}$. Il apparaîtrait au contraire plus équitable d'établir une péréquation des moyens recueillis par les établissements à l'échelon le plus large possible. Faute de quoi nous constaterons, comme aux Etats-Unis, " des abandons précoces de jeunes de milieux modestes ou discriminés, pour lesquels le risque de l'endettement est le plus élevé " (Vinokur [2009, p. 445]) et un accroissement des inégalités de revenus (à diplôme égal), lié aux inégalités face au poids de la dette. Dans cette perspective, il conviendrait aussi d'étudier - mais cela dépasse le cadre de notre article - les cercles vertueux qui peuvent s'établir en faveur des meilleurs établissements (toujours plus attractifs, plus riches et donc en mesure d'accorder des aides) au détriment d'autres établissements, victimes d'un effet contraire.

Vinokur [2009] propose donc de penser un système de tarification des études supérieures au niveau national, garantissant notamment l'égalité de traitement, l'accessibilité indépendamment des origines sociales et géographiques et la transparence. Pour cet auteur, le barème des frais d'inscription et des aides doit donc dépendre des revenus des parents. Il doit aussi être identique sur l'ensemble du territoire pour toutes les filières et voté en même temps que le budget de l'enseignement supérieur, dans le cadre d'une programmation pluriannuelle. Cette approche pose néanmoins des problèmes : d'une part parce que les étudiants restent sensibles à l'endettement, et ce de manière différente selon la classe sociale (voir la troisième section de l'article), d'autre part parce qu'elle construit les conditions politiques d'une renonciation au service public de l'enseignement supérieur à travers la libéralisation progressive des frais d'inscription, une fois ceux-ci instaurés.

\section{Le point de vue de l'efficience}

Les partisans de frais d'inscription élevés s'appuient aussi sur l'argument néolibéral de la "supériorité des marchés " (Johnstone [2004]) : en libéralisant les frais d'inscription, la concurrence sur 
le marché de l'enseignement supérieur est supposée permettre de refléter les coûts et les avantages relatifs, en termes de débouchés, de chaque formation. Chaque individu déciderait de son investissement éducatif de manière rationnelle. L'équilibre général conduirait ainsi à révéler la valeur implicite des formations en intégrant les bénéfices attendus et les risques encourus par chacun (individus, entreprises et collectivité). Dans le cadre de cette approche théorique, le fonctionnement marchand favoriserait d'une part l'efficience allocative, en agissant comme un mécanisme de sélection et d'orientation, et, d'autre part, l'efficience productive en poussant à l'effort les étudiants, les enseignants et les universités.

\section{La sélection}

La question de la "sélection des talents" est un des piliers de l'économie de l'éducation depuis les travaux de Friedman [1962]. Gary-Bobo et Trannoy [2008] prolongent cette approche et considèrent les frais d'inscription comme un moyen de trier efficacement les étudiants. Selon ces auteurs, les étudiants disposent en effet d'une information imparfaite sur leurs propres talents et imparfaitement observable par les universités. L'asymétrie d'information conduit à un phénomène d'opportunisme ex ante (anti-sélection) que des frais d'inscription suffisamment élevés permettraient de combattre. Si l'on considère que les examens ne servent que de révélateur d'une information privée ${ }^{7}$, une sélection par des examens apparaîtrait de surcroît coûteuse pour les universités qui doivent les organiser et coûteuse pour les étudiants, contraints à " gaspiller" des ressources (pécuniaires et non pécuniaires) dans la préparation de leurs examens. Les frais d'inscription seraient alors un substitut préférable aux examens d'entrée comme outil de sélection des étudiants.

Ces modèles de sélection décentralisée fondés sur la liberté des frais d'inscription, souffrent cependant de sérieuses faiblesses. Ils reposent sur l'hypothèse selon laquelle l'étudiant s'inscrit si et seulement si les gains escomptés de son passage par l'enseignement supérieur sont supérieurs au coût des études, 
se comportant " en bon statisticien (calculant) ses chances de gagner tel ou tel salaire demain en fonction des estimations de son talent"(Gary-Bobo et Trannoy [2005, p. 201]).

Dans la réalité, les individus ne disposent d'une information suffisante ni sur leur propre compte, ni sur le système universitaire, ni même sur les débouchés et les perspectives d'évolution du marché du travail. La rationalité des étudiants est donc fondamentalement limitée par leur capacité à disposer des informations nécessaires et à les traiter. En outre, on sait, depuis les travaux de Bourdieu, que l'origine sociale influence fortement les choix de parcours des étudiants en renforçant la reproduction sociale : " les étudiants sont d'autant plus modestes dans leurs ambitions scolaires (comme d'ailleurs dans l'évaluation de leurs résultats) et d'autant plus bornés dans leurs projets de carrière qu'ils appartiennent à des catégories dont les chances scolaires sont les plus faibles " (Bourdieu [1974], p. 9). Selon cette " causalité du probable ", "le fait d'avoir des chances positives ou négatives d'être, d'avoir ou de faire quelque chose (prédispose), en prédestinant à agir en sorte que ces chances se réalisent " (Bourdieu [1974, p. 28]). La stratégie éducative des familles fait ainsi partie d'un "système de stratégies de reproduction » et d'une maximisation d'un " rendement symbolique ".

Des conclusions similaires sont exprimées dans les travaux de Boudon [1974, 1994] ou dans les travaux et la revue de littérature de Haveman et Wolfe [1995]. Elles ont par la suite été théorisées et testées empiriquement (par exemple Micklewright [1989] pour l'influence du type d'emploi des parents ou Hearn [1991] pour celle du revenu). Sullivan [2006] montre ainsi empiriquement que "le contexte social et le sexe des élèves ont un impact sur la perception qu'ont les étudiants de leurs propres capacités " (p. 1). Ce résultat est cohérent de surcroît avec les erreurs cognitives systématiques identifiées ou suggérées par les psychologues sociaux ${ }^{9}$.

Les décisions des étudiants en fonction de leur milieu social d'origine ont également été modélisées par la théorie de l'aversion relative au risque ${ }^{10}$ (ARR) qui distingue ainsi deux 
types d'effets de l'origine sociale sur l'éducation. Les effets principaux, d'une part, reposent sur l'impact des capacités cognitives des étudiants (fondées principalement sur les ressources économiques et culturelles disponibles). Des effets secondaires, d'autre part, sont liés à l'impact du milieu social pour des individus ayant les mêmes capacités cognitives. En suivant Boudon, la théorie de l'aversion relative au risque établit que ces effets secondaires peuvent s'expliquer par la différence entre gains d'utilité pour un même choix éducatif : la crainte d'un déclassement social serait supérieure au désir d'ascension sociale ${ }^{11}$. L'ambition scolaire dépendrait donc de la position sociale de départ de chaque individu, ce que confirment empiriquement Holm et Jaeger [2008].

Dès lors, en modélisant cette hétérogénéité sociale par un biais d'auto-dévalorisation de leurs talents par les individus issus de milieux défavorisés, Flacher et Harari-Kermadec [2013] reconsidèrent les résultats de Gary-Bobo et Trannoy [2008], en montrant que les frais d'inscription présentent une double inefficacité pour sélectionner les étudiants : ils tendent à éliminer de bons étudiants qui auraient " mérité » d'entrer à l'université mais ont sous-estimé leurs talents et ils tendent à intégrer des étudiants médiocres ayant bénéficié de conditions familiales favorables. Des frais d'inscription suffisamment faibles apparaissent donc être une condition clef, nécessaire bien que non suffisante, pour atteindre un équilibre socialement optimal dans ce cadre théorique.

Enfin, cette conclusion apparaît renforcée par le caractère vraisemblablement endogène et dynamique des capacités cognitives des individus : si celles-ci ne sont pas simplement " révélées " par l'éducation mais aussi "développées " par elle, les frais d'inscription devront être d'autant plus bas qu'ils devront se garder de filtrer une population initialement peu " douée " mais dont l'éducation accroît le potentiel.

\section{Orientation des étudiants et marché du travail}

Pour les partisans des frais d'inscription, ceux-ci pourraient servir à orienter les étudiants dans le choix d'un cursus et finale- 
ment d'un débouché professionnel. Des frais d'inscription ou des subventions différenciées entre cursus permettraient ainsi de favoriser les cursus jugés les plus utiles socialement et donc d'internaliser les externalités de l'éducation et de corriger les défaillances de marché. Cependant, cette logique suppose, a minima, qu'étudiants potentiels, universités et pouvoirs publics disposent d'une information complète et fiable sur les formations, leurs débouchés, leur valeur à long terme, leur utilité sociale ainsi que sur les risques liés à l'évolution de la conjoncture économique. Cette connaissance ne doit pas se limiter au court terme mais intégrer les perspectives d'évolution à long terme de ces différentes variables. Les étudiants doivent être mobiles géographiquement et bénéficier de conditions leur permettant de réaliser leurs études indépendamment des ressources de leurs parents (bourses, accès au crédit...). Enfin, les cursus doivent être relativement substituables entre eux. Ces conditions sont évidemment peu réalistes.

Les frais d'inscription et l'endettement associé pourraient, dans les faits, transformer la nature des relations à l'emploi, en particulier pour les étudiants dont le bagage culturel ou social pousse à des études courtes ou professionnalisantes (Cameron et Heckman [2001]) plutôt qu'à une prise de risques éducatifs. Les emplois publics pourraient être délaissés du fait de leur rémunération inférieure (Field [2009]) et occupés par des diplômés des établissements les moins prestigieux.

L'effet d'orientation et la dette pèsent donc sur certaines classes sociales et essentiellement dans la perspective de répondre aux exigences du marché du travail : les étudiants seront contraints d'entrer très vite dans la vie active à la fin de leurs études, voire d'accepter des emplois ne correspondant pas à leur qualification, pour assumer les remboursements auxquels ils se sont engagés. L'instauration d'un marché éducatif contribue à asservir les futurs travailleurs, à travers le poids de la dette qui repose sur eux, poussant certains à s'exiler vers des pays offrant des salaires plus élevés (Vinokur [2009]). 


\section{Les incitations à l'effort}

Le rendement de l'éducation dépend notamment de l'implication des étudiants dans leurs études, du temps et de l'attention que les enseignants peuvent porter à chaque étudiant et des efforts de l'université à proposer les formations et conditions d'accueil les plus propices.

Or, « il y a des incitations vraisemblablement bien plus élevées de la part des étudiants à travailler dur et à obtenir leur diplôme "dans les temps" (Johnstone [2004], p. 407) ou à mieux choisir leur cursus (Gregoir [2008]) lorsqu'ils payent leurs études. Selon Gary-Bobo et Trannoy [2005] et Gregoir [2008], en présence de frais d'inscription élevés, l'étudiant exercerait également une pression sur ses enseignants et son université dont il attend, en tant que " client ", le meilleur rapport qualitél prix $^{12}$. Les frais d'inscription permettraient donc de réduire le risque moral bilatéral (celui, pour l'étudiant, de se laisser aller et celui, pour l'enseignant et l'université, de se montrer complaisant dans l'évaluation) et d'accroître « la pression du marché du travail sur les équipes enseignantes et sur les étudiants " (GaryBobo et Trannoy [2005, p. 206]).

Ces arguments, peu étayés empiriquement, sont contestables : dans les faits, l'absence de frais d'inscription ne signifie pas que l'étudiant se désintéresse de sa formation. Il suffit, pour s'en convaincre, de se représenter l'exemple des classes préparatoires dans le contexte français. Ils négligent de plus les coûts indirects (d'entretien et d'opportunité) qui pèsent lourd sur la population étudiante. Enfin, le ressort de la motivation des étudiants comme des enseignants (et chercheurs) n'est pas, en premier lieu, une question financière : la reconnaissance par les pairs et la relation étudiant/enseignant peuvent constituer des moteurs bien plus puissants de la motivation qu'une rémunération dépendant du niveau de réalisation d'objectifs pré-assignés ou qu'une relation de client à fournisseur. Il en va de même des meilleurs établissements français dont la réputation et les efforts ne sont fondés jusqu'ici ni sur une maximisation de leurs " pro- 
fits ", ni sur des mécanismes marchands, ni sur un marketing coûteux ${ }^{13}$.

\section{Le financement du système éducatif}

Des besoins financiers...

La troisième catégorie de raisons invoquées pour légitimer les frais d'inscription repose sur le nécessaire financement de l'enseignement supérieur. Cet argument est notamment utilisé dans les pays (comme la France) dans lesquels les subventions publiques (ou privées) apparaissent insuffisantes pour atteindre un niveau de dépense par étudiant similaire à celui des pays de l'OCDE les plus avancés en termes de taux de diplômés. Ce niveau est cependant sujet à débats et est difficile à établir dans la mesure où il dépend des filières éducatives, du choix d'orientation des étudiants entre ces filières, des choix pédagogiques, du niveau des salaires des enseignants dans le pays considéré, des trajectoires institutionnelles nationales, etc.

La France consacrait ainsi, en 2008, 1,4\% de son PIB à l'enseignement supérieur (dont 1,2\% provenant des dépenses publiques) alors que les Etats-Unis en consacrent 2,7\% (1\%), le Canada 2,5\% (1,5\%), le Royaume-Uni $1,2 \%(0,6 \%)$ et l'OCDE en moyenne $1,5 \%$ (1\%). Ces différences, dans des pays aux démographies relativement comparables, se traduisent par des écarts importants en matière de dépenses par étudiant : en moyenne, celles-ci sont de 14079 USD en France contre 29910 USD aux Etats-Unis, 20903 USD au Canada et 15310 USD au Royaume-Uni, pour une moyenne de 13717 USD dans les pays de l'OCDE. Or ces disparités dans les niveaux des dépenses pour l'enseignement supérieur semblent liées au taux de diplômés du supérieur (en particulier pour les études les plus longues), légitimant l'exigence d'un financement accru du système français et européen d'éducation. Selon l'OCDE [2010], le taux de diplômés de l'enseignement supérieur pour les 25/64 ans en 2006 est en effet de 39,5\% aux Etats-Unis et $47 \%$ au Canada 
contre 26,2 \% en France et 30,5\% en Angleterre (OCDE [2010] et OCDE [2011], pp. 234 et 248 respectivement).

Cependant, le fait que le niveau moyen des dépenses en France soit proche du niveau moyen constaté au sein de l'OCDE (en pourcentage du PIB) ne doit pas masquer les importantes disparités de financement entre établissements et entre filières. La France se caractérise en effet par un dualisme très marqué de son enseignement supérieur : en 2007 le coût par étudiant variait de 8970 euros à l'université contre 13880 euros en classes préparatoires aux grandes écoles ${ }^{14}$. Les différences de dépense publique par étudiant, en fonction du diplôme, sont encore plus impressionnantes puisque, selon Courtioux [2009], si les dépenses publiques s'élèvent à 37294 euros, en moyenne, pour un diplôme à $\mathrm{Bac}+5$, elles sont de seulement 17805 euros pour un étudiant de Master contre 127527 euros pour un diplômé d'une "très grande école d'ingénieur " (pour la génération de 1970, année de référence : 2005). Il résulte de ces données un relatif consensus selon lequel l'université française est sous-financée et selon lequel un effort particulier doit être réalisé (CERC [2003], Trannoy [2006]).

De ce point de vue, la situation de l'enseignement supérieur français n'est pas sans faire penser au relatif échec de la "stratégie de Lisbonne " qui visait 2\% du PNB en dépenses d'éducation en 2010. Pour la Commission européenne, "si on a assisté à une croissance bienvenue des effectifs étudiants, celle-ci n'a pas été accompagnée par une hausse corrélative des financements publics et, en Europe, les universités n'ont pas eu la possibilité de compenser ce déficit par un recours à davantage de fonds privés. [...] Il est probable qu'à l'avenir la plus grande part des ressources nécessaires pour mettre fin au sous-financement des universités devra provenir de sources non publiques " (Commission européenne [2006] p. 4).

\section{... que les frais d'inscription ne peuvent/doivent pas combler}

Si une hausse de la fiscalité est (trop) souvent écartée, les frais d'inscription sont parfois considérés comme un moyen d'ac- 
croître non seulement les ressources des établissements mais également les budgets qui servent à l'octroi de bourses. En Europe, les pratiques en la matière varient sensiblement, d'un financement entièrement public sans frais d'inscription (Suède, Finlande et Danemark), à l'instauration de frais d'inscription pouvant représenter moins de $6 \%$ du budget des étudiants dans certains pays (Autriche, Malte, République Tchèque, France, Norvège), entre 7 et $15 \%$ du (Pologne, Slovaquie, Espagne, Croatie, Portugal, Pays-Bas, Estonie), voire 17 à $24 \%$ de leur budget total (Royaume-Uni, Lettonie, Irlande, Turquie) ${ }^{15}$.

Cependant, si nous admettons la réalité des besoins financiers, la solution consistant à relever les frais d'inscription n’apparaît pas nécessairement pertinente. D’abord, les financements ne s'accroissent significativement qu'à la condition que l'Etat ne se désengage pas, parallèlement à la hausse des frais d'inscription. Or cette crainte n'est pas sans fondement ${ }^{16}$ :

- certains Etats, à l'image du Royaume-Uni lors de la réforme de 2010, ont d'emblée annoncé leur désengagement partiel (80\%) du financement récurrent des universités ;

- les mécanismes d'accompagnement des politiques de frais d'inscription (en particulier les prêts à remboursement conditionnel) peuvent se révéler très coûteux pour la collectivité (voir la deuxième section de cet article). L'Etat peut alors être amené à réallouer ses fonds vers ces mécanismes, au détriment des dotations aux universités ;

- l'incitation à se désengager du financement récurrent des établissements mais aussi des bourses sera d'autant plus forte que l'accès au crédit permettra d'accroître les frais d'inscription et de donner aux étudiants une apparence d'autonomie par le crédit (Johnstone [2004]). De plus, le financement des aides aux étudiants, surtout s'il repose sur des mécanismes locaux, sera particulièrement réduit dans la mesure où les universités bénéficient d'un accroissement d'autant plus important de leurs ressources que la redistribution est limitée (au risque de restreindre fortement l'accès des classes populaires aux bonnes universités). Il existe alors, au sein des établissements, un arbitrage entre accroissement des ressources et démocratisation de l'accès. 
Ensuite, les financements pourraient ne servir que partiellement à l'amélioration du système d'enseignement supérieur : les recettes seront pour partie absorbées par des dépenses de marketing, des dépenses somptuaires ou de lobbying, par des projets d'expansion ou par le recrutement d'enseignants et de chercheurs dont le coût sera lié à la renommée ${ }^{17}$. Or il n'est pas démontré, à notre connaissance, que ces dépenses contribuent à une amélioration de la qualité générale de l'enseignement supérieur. De surcroît, dans un contexte de concurrence monopolistique dans lequel l'élasticité de la demande est faible et dans lequel le prestige de l'établissement joue un rôle clef, l'appétit pour de nouveaux financements n'apparaît pas bornée. D’après les chiffres cités par Vinokur [2009], p. 445, la dépense d'instruction par étudiant et celle de l'administration, corrigée de l'inflation, ont respectivement augmenté de $17 \%$ à $54 \%$ entre 1960 et 2001 dans les établissements publics d'enseignement supérieur aux Etats-Unis. Pour les ménages américains, entre 1975 et 2005, le coût annuel de la scolarité dans les collèges publics est passé de 10 à 21 semaines de salaire moyen (de 21 à 53 semaines dans les collèges privés).

\section{Frais d'inscription et mécanismes d'accompagnement : atouts et limites}

\section{Des mécanismes de compensation financière sont néces- saires : aides directes et indirectes}

Lorsqu'un étudiant issu d'un milieu modeste a des capacités cognitives suffisantes, une condition nécessaire (bien que non suffisante) pour pouvoir entreprendre des études réside dans la possibilité d'accéder à des ressources financières (principe de compensation). Ces ressources doivent couvrir les frais d'inscription et les frais d'entretien ${ }^{18}$. Cette condition est particu- 
lièrement importante pour éviter aux étudiants d'avoir à assumer une activité rémunérée pour payer leurs études. En effet, une telle activité, d'une part, réduit le temps disponible pour les études (accroissant de ce fait le taux d'échec - cf. Eckstein et Wolpin [1999]) et, d'autre part, tend à fermer les perspectives scolaires les plus prestigieuses (car ce sont souvent les plus chronophages). Au-delà des moyens que les familles peuvent consacrer aux études de leurs enfants, il s'agit donc de mettre en place des mécanismes d'accompagnement : des aides directes (bourses, exonérations fiscales) ou indirectes (en offrant un accès au crédit).

\section{Les aides directes : bourses et exonérations fiscales}

Les étudiants peuvent bénéficier de bourses, d'aides spécifiques (notamment en matière de logement), d'exonération de frais d'inscription ou, via leur famille, d'exonérations fiscales. Ces aides peuvent être universelles ou conditionnées. Dans ce dernier cas, elles peuvent dépendre du revenu des parents ou du " mérite " de l'étudiant, autrement dit de ses résultats académiques.

En France, les aides sur critères sociaux restent limitées et insuffisantes (en montant et en assiette) pour corriger l'effet de l'origine sociale sur l'accès à l'enseignement supérieur ${ }^{19}$. D’après les chiffres cités par Trannoy [2006], les aides octroyées aux étudiants pesaient, en 2003, 0,2\% du PIB contre 0,6\% en Suède, $0,5 \%$ au Royaume-Uni et $0,4 \%$ aux Pays-Bas. Les bourses, en particulier, ne suffisent pas à lever la contrainte qui pèse sur beaucoup d'étudiants d'avoir à travailler pour financer leurs études. Il serait possible d'avancer que ces bourses limiteraient l'auto-sélection et maintiendraient donc des étudiants de niveau trop faible dans l'enseignement supérieur. Un arbitrage symétrique entre équité et efficience peut exister avec des bourses "au mérite": ces bourses se concentrent sur les meilleurs étudiants et les incitent à l'effort. Cependant, les étudiants ayant les meilleurs résultats sont aussi ceux qui proviennent des classes sociales les plus favorisées. L'octroi de bourses au mérite 
reste donc relativement peu équitable. Le croisement des critères de mérite et de critères sociaux ne résout que partiellement le problème, dans la mesure où le système éducatif peine à valoriser les talents, pourtant réels, issus des milieux populaires. Les exonérations fiscales, pour leur part, cumulent les défauts en étant à la fois potentiellement inefficientes et particulièrement inéquitables puisqu'elles concernent les milieux les plus favorisés et pèsent particulièrement lourd au regard des aides attribuées selon le critère social. Le système d'aide dans l'enseignement supérieur apparaît finalement anti-redistributif puisqu'il bénéficie davantage aux classes favorisées par le biais des dépenses collectives et par les forts avantages fiscaux octroyés aux ménages les plus aisés ${ }^{20}$ (Colin [2008], p. 6).

Enfin, plusieurs auteurs justifient des aides aux étudiants pour compenser les effets distorsifs de la fiscalité progressive sur le revenu. En effet, Dupor et al. [1998] ont montré que la progressivité de cette fiscalité dans les années 1970 avait conduit à une baisse de l'investissement en capital humain. De même, Sturn et Wohlfahrt [2000] montrent qu'en raison de cette progressivité, les diplômés paieraient plus d'impôts que les nondiplômés et percevraient les mêmes gains nets sur leur cycle de vie. C'est dans cette perspective que Barbaro [2005] modélise le revenu sur le cycle de vie et montre ainsi qu'une subvention à l'éducation compensant les effets de la fiscalité progressive sur le choix des études serait efficiente dans la mesure où, sans inciter les individus peu doués à faire des études, elle éviterait à des étudiants relativement doués d'y renoncer (voir aussi Bovenberg et Jacobs [2005]). De surcroît, le modèle de Barbaro [2005] est construit de sorte que cette solution serait relativement équitable car elle permettrait d'accroître le revenu net sur le cycle de vie de l'ensemble des groupes d'individus. Il est cependant permis de douter, au-delà du simple problème d'information et au regard des biais psycho-sociaux présentés plus haut ainsi que des déterminants des choix individuels (qui ne se limitent pas aux questions de revenu), que les individus soient en mesure de raisonner efficacement sur l'ensemble de leur cycle de vie, avec une estimation juste de leurs revenus, de la fiscalité et de leurs évolutions. 


\section{L'accès au crédit : pilier de l'éducation par capitalisation}

Un étudiant issu des classes populaires pourra-t-il recourir à l'emprunt ? Sera-t-il obligé, au contraire, de travailler pendant ses études ? Préferera-t-il les abandonner ou choisira-t-il des filières plus courtes et moins prestigieuses? Du fait d'externalités positives liées à l'éducation, le coût pour la collectivité de voir un individu ne pas entreprendre des études alors qu'il en a les capacités justifie une intervention publique. Indépendamment des aides directes, rendre le crédit accessible constitue une option politique, théorisée par Friedman [1962] pour compenser les inégalités de ressources, et privilégiée aux aides directes dans une partie de la littérature (Barr [1993], Gary-Bobo et Trannoy [2005], Gregoir [2008], Johnstone [2004], Trannoy [2006]). Le crédit permettrait en effet à n'importe quel étudiant suffisamment talentueux, indépendamment de son milieu social, de s'adapter aux frais d'inscription demandés par les universités. Le crédit peut ainsi devenir le pilier d'une "éducation par capitalisation ", en visant l'accumulation du capital humain.

Cependant, les rendements de l'éducation sont risqués : d'abord, ils reposent sur un capital humain qui, par nature, est immatériel et non hypothécable. Ensuite, ces rendements espérés sont soumis à des risques de vie (principalement des problèmes de santé) et à des problèmes d'orientation : l'étudiant connait-il suffisamment bien la voie dans laquelle il s'engage et ses débouchés ? Est-il capable de réussir ? Sera-t-il toujours aussi motivé par son choix ? De plus, le prêteur peut être victime d'un risque d'anti-sélection lors de la signature du prêt (si l'emprunteur ne fournit pas toutes les informations dont il dispose sur sa capacité à rembourser) et d'un aléa moral dans la phase de remboursement (si l'emprunteur ne fait pas les efforts nécessaires pour tenir ses engagements). Enfin, les gains attendus sont aussi soumis aux transformations structurelles et aux évolutions conjoncturelles de l'économie, conduisant les banques à privilégier les étudiants des écoles les plus prestigieuses, au détriment des étudiants à revenu moyen ou modeste, sous-représentés dans ces filières, mais qui ont le plus besoin de recourir à l'emprunt. 
Ainsi, dans un marché financier imparfait, les projets de formation dont la rentabilité privée est positive ne peuvent pas tous être financés par l'emprunt. C'est pour répondre à cette limite que, dans la lignée des travaux de Friedman, des formules de prêts subventionnés ont été proposées.

\section{Les prêts à remboursement conditionné au revenu (PARC)}

Les PARC sont des prêts dont le remboursement dépend du revenu courant des individus ayant terminé leurs études et se trouvant en position d'activité. Ils ont été proposés par Friedman en 1955. Leur principe repose, d'une part, sur l'idée qu'il serait raisonnable de faire payer le bénéficiaire d'une formation dans la mesure où il en tirera un avantage tout au long de sa vie et, d'autre part, sur la nécessité de compenser, par une intervention publique, le sous-investissement qui résulte des externalités positives liées à l'éducation. C'est ainsi que, pour Friedman ([1962], p. 103), "la contrepartie à l'éducation pourrait être d' " acheter " une part des perspectives de revenus futurs de l'individu (et) de lui avancer les fonds dont il a besoin pour financer sa formation ".

Ce système de quasi-titrisation du capital humain a été expérimenté à l'université de Yale avant de s'étendre progressivement à plusieurs pays (dont la Suède, l'Australie, le Chili, la Nouvelle-Zélande, le Royaume-Uni...). Il est envisageable selon différentes modalités : les prêts peuvent être accordés par l'Etat ou par des banques commerciales, bénéficiant de garanties publiques ; l'Etat peut définir les conditions d'éligibilité à ces prêts, leurs plafonds (doivent-ils couvrir le coût de la formation ou les frais d'entretien ?), leurs taux et bonifications, la durée et les modalités de remboursement, les règles en cas de non-obtention du diplôme, de réorientation ou de difficultés de remboursement, la progressivité éventuelle des remboursements ou une différenciation des aides entre formations ${ }^{21}$; l'Etat peut également décider du niveau de mutualisation des risques (au plan national ou entre anciens étudiants). En utilisant ces différents leviers, les PARC permettent de définir différents équilibres en 
termes de partage des gains et des risques entre la collectivité et l'étudiant.

Contrairement aux prêts hypothécaires, en mutualisant les risques et en bénéficiant de garanties de l'Etat, les PARC traiteraient de manière équitable les potentiels des candidats indépendamment de leur milieu social d'origine (Bennett et al. [1992]) : l'étudiant, une fois dans la vie active, rembourse en fonction de ses capacités contributives réelles. Les PARC présentent aussi l'avantage de ne pas obérer l'avenir bancaire d'un individu qui aurait fait défaut auprès de sa banque (Dynarski [1994]), en ne l'excluant pas de futurs prêts. Ils réduisent donc les risques de voir un étudiant renoncer à ses études du fait de leur coût. Ils améliorent les conditions de vie des personnes concernées en leur permettant de lisser leur consommation entre des années plus fastes et des années qui le sont moins, et tiennent ainsi compte de la capacité de paiement des étudiants sur leur cycle de vie (Vandenberghe et Debande [2008]). Par conséquent, les PARC favoriseraient l'équité, et ce d'autant plus qu'ils permettraient de couvrir l'ensemble des frais d'inscription et des coûts d'entretien de l'étudiant et qu'ils mettraient à contribution des individus s'installant à l'étranger après leurs études.

Néanmoins, cette équité concerne avant tout le principe de compensation (financière). Elle ne garantit pas l'équité contributive, puisque celle-ci dépend du degré de progressivité introduit dans les PARC et que, dans les faits, la contribution apparaît souvent simplement proportionnelle au revenu (Vandenberghe et Debande [2008]). En outre, elle ne constitue qu'une liberté formelle d'accéder aux études mais en aucun cas une liberté positive, notamment dans les milieux sociaux qui ne prédisposent pas aux études. Kane [1994] montre ainsi que, dans les années 1980 aux Etats-Unis, les classes sociales défavorisées voient leur accès à l'université baisser du fait de la hausse des frais d'inscription. Si le taux de scolarisation de ces classes sociales a finalement augmenté, c'est avant tout parce que les enfants issus de ces classes ont bénéficié du fait d'avoir des parents mieux éduqués (car ayant bénéficié d'importantes opportunités d'accéder à l'éducation dans l'après 
seconde-guerre). Alors que les " prêts garantis » ne représentent qu'une "solution partielle à la contrainte d'endettement, dans la mesure où il y a des limites au montant que l'étudiant peut emprunter "(Kane [1994, p. 880]), nous verrons également que la contrainte d'endettement, et surtout le poids de la dette, posent des problèmes et que ces problèmes pèsent davantage sur les classes populaires.

Ensuite, en permettant le développement des frais d'inscription, les PARC contribueraient à accroître l'efficience, et ce d'autant plus qu'ils n'affecteraient pas significativement le taux de rendement privé des diplômés (ce qui suppose, selon Vandenberghe et Debande [2008], de prendre en compte l'antisélection qui peut résulter d'une mutualisation des risques entre populations aux perspectives professionnelles hétérogènes). Or, d'un point de vue théorique, en assurant le risque individuel (l'individu peut se soustraire aux remboursements en cas d'échec professionnel), les PARC réduisent, voire suppriment, l'effet sélectif des frais d'inscription et aucun individu n'a intérêt à renoncer à ses études. Cette option accroît donc théoriquement l'anti-sélection (choix inadapté de la formation) et l'aléa moral (absence d'effort d'insertion dans la vie active ${ }^{22}$ ). Il deviendrait alors nécessaire d'établir des mécanismes de sélection (au moins pour les filières les plus coûteuses) dont nous savons par ailleurs qu'ils ne sont pas socialement neutres (Bourdieu [1974]). Dans le cas où les PARC ne couvrent que partiellement le risque individuel, en plafonnant le montant du prêt au-dessous du coût total effectif de la scolarité ou en durcissant les conditions de remboursement, les frais d'inscription redeviennent sélectifs. Cependant, des étudiants issus des classes populaires sont alors susceptibles de renoncer à leurs études, de réduire leurs prétentions ou de modifier le choix de leur cursus, conduisant à un sous-investissement éducatif. Il existe ainsi un arbitrage entre efficience et équité. Enfin, des frais d'inscription substantiels permis par les PARC n'accroissent pas nécessairement les ressources d'un établissement (voir la section précédente) et ce d'autant plus que l'anti-sélection et l'aléa moral peuvent rendre les PARC coûteux : en amont, les étudiants les moins 
sûrs d'être en mesure de rembourser auront tendance à recourir aux PARC, alourdissant ainsi le poids fiscal du mécanisme. Inversement, les étudiants les plus doués et motivés préfèreront ne pas participer à ce système de prêt s'ils sont contraints de financer la mutualisation des risques. En aval, les plus doués et motivés auront intérêt à réduire leurs efforts et à tricher sur leur revenu pour réduire leurs remboursements. De leur côté, les banques tendront à réduire leurs efforts de recouvrement, augmentant ainsi le poids de ces prêts sur les finances publiques. A moins de n'assurer le risque que de manière très limitée (et donc de décourager la poursuite d'études), les PARC peuvent donc peser sur les comptes publics et profiter davantage au secteur financier ${ }^{23}$ qu'aux universités.

Certaines solutions peuvent partiellement atténuer ces limites : opérer une sélection des étudiants à l'entrée des formations, définir des plafonds de contribution (en cas de mutualisation des risques entre emprunteurs), limiter la durée d'étude pour laquelle il est possible d'emprunter et la moduler en fonction du degré de réussite, échelonner le versement du prêt, développer différents contrats de PARC, etc. Trannoy [2006] propose également une titrisation de la dette étudiante. Il considère en effet les bénéfices d'une mutualisation du risque en comparant PARC et options financières. Le seuil de revenu qui déclenche le remboursement du PARC serait assimilable au seuil d'une option sur le capital humain d'un individu à partir duquel l'exercice de cette option est profitable. L'auteur évoque alors la possibilité de développer un marché de ces options, si l'Etat ou les banques ont besoin de fonds. Dans la même veine, Palacios [2004] propose une solution purement privée de " contrats en capital humain ", sorte de prise de participation dans la réussite de l'étudiant. Ce mécanisme, assimilable à une titrisation d'une partie des revenus futurs de l'étudiant, conduit l'investisseur à financer la formation initiale en échange d'une fraction du revenu pendant une durée définie contractuellement. Il nécessite néanmoins de trouver les investisseurs (ce qui revient à poser la question de l'accès au crédit). Les risques associés à ces titrisations ne semblent cependant pas avoir été 
mesurés au regard de ce que nous savons de ces mécanismes depuis la crise des "subprimes $"{ }^{24}$. Ces différentes "solutions" visant à favoriser les PARC sont donc partielles et discutables. Elles ne constituent généralement que le résultat d'un arbitrage entre objectifs contradictoires.

\section{Suffit-il de desserrer la contrainte d'endettement ? Origine sociale, accès au crédit et poids de la dette}

La réalité de la contrainte d'endettement, ainsi que son poids (relativement à d'autres variables explicatives), sont discutés dans la littérature. C'est ainsi que les travaux de Frenette [2007] sur des données canadiennes ont conclu à une importance limitée de cette contrainte. Ces résultats sont cohérents avec ceux de Carneiro et Heckman [2002] ou de Keane et Wolpin [2001]. Ce serait en effet la nature des transferts entre parents et enfants (à travers le soutien scolaire, notamment) de même que le niveau d'études des parents, leurs attentes, la qualité de l'établissement secondaire ou les contraintes financières qui expliqueraient, bien plus qu'une éventuelle contrainte d'endettement, l'inscription et la réussite universitaire ${ }^{25}$.

De surcroît, même si la contrainte d'endettement existe pour les plus défavorisés (Plug et Vijverberg [2005]), desserrer la contrainte d'endettement ne remet pas en cause les objections mentionnées dans la section précédente. Faciliter l'accès au crédit ne suffit pas à contrebalancer la " causalité du probable " qui, conséquence des déterminismes sociaux, pousse les individus les moins favorisés à ne pas croire dans leur potentiel éducatif. Cela ne suffit pas non plus à résoudre les problèmes de financement des universités.

En outre, même dans le cadre de PARC, la dette pèse sur les orientations universitaires et les choix de carrière de tous les étudiants et de manière plus aiguë sur ceux issus d'un milieu modeste : il est en effet contestable de considérer équivalent, dans la décision de poursuite d'études, de bénéficier d'un environnement familial en mesure d'en financer le coût, 
et de bénéficier d'un " droit à emprunter ». Des publications insistent en effet sur le poids de la dette et son impact bien réel sur les choix de la filière et sur la nature des métiers exercés à l'issue du cursus (fonction publique ou secteur privé, par exemple). A partir d'une expérience réalisée sur les étudiants d'une université, Field [2009] montre empiriquement que les comportements d'emprunt et de réaction au fardeau de la dette ne sont pas rationnels. En outre, les choix de carrière apparaissent distordus au profit d'emplois rémunérateurs et au détriment d'emplois qui auraient pu être socialement plus utiles $^{26}$. Inversement, pour Field ([2009], p. 19), « du point de vue du bien-être social, la réduction de la dette peut accroître l'investissement éducatif débouchant sur des emplois à rendements sociaux élevés ». Callender [2006] montre également que l'aversion à la dette décourage les étudiants des familles à bas revenus. Pour sa part, Frenette [2005] met en évidence que la classe moyenne en Ontario a vu sa probabilité de poursuivre des études fortement diminuer du fait de la hausse soudaine des frais d'inscription. Des résultats similaires sont obtenus par Brodaty et al. [2009] : une hausse des frais d'inscription apparaît relativement plus dommageable à l'intégration, dans le système éducatif, des enfants issus des classes sociales les moins éduquées, ce qui peut s'expliquer, selon Orfield [1992] ou Pennell et West [2005], par une élasticité au revenu de la demande d'enseignement supérieur plus importante dans la tranche inférieure des revenus. On observe ainsi aux Etats-Unis " une tendance à la bi-polarisation de la demande : abandons précoces pour les uns, forte croissance de la demande (surtout qualitative) pour les autres"(Vinokur [2007], p. 225), les étudiants originaires de milieux modestes regrettant davantage leur endettement (contrairement à une majorité de ceux issus de milieux aisés qui le considère bénéfique). Il peut alors paraître erroné de considérer qu'un accès " de droit " au crédit pour financer des études universitaires permet un accès effectif à ces cursus et donc à l'emprunt (Bourdieu [1974], pp. 15-16, Sen [2010]). 


\section{Conclusions et perspectives}

Alors que l'opportunité de développer une tarification des études supérieures se fait jour dans de nombreux pays, et notamment en France, notre article pose les différents éléments du débat. Nous avons ainsi montré que les frais d'inscription, de même que la quasi-gratuité de l'accès à l'enseignement supérieur, ne permettaient pas, seuls, de garantir un modèle d'enseignement supérieur équitable, efficient et efficace en termes de financement, ou de s'en approcher.

Nous avons pour cela défini l'équité comme la conjonction d'un principe de compensation financière, d'un principe d'équité contributive et de libertés positives. Nous avons rappelé, à cet égard, les limites du système d'enseignement supérieur français qui, du fait de la conjonction de sa quasi-gratuité, d'une sur-représentation des classes favorisées et de bourses insuffisantes, est inéquitable. Pour autant, nous avons également montré que la mise en place de frais d'inscription ne permettait pas nécessairement de rétablir l'équité : il faudrait, pour cela, que les contributions soient progressives et que les compensations financières en direction des classes moins favorisées soient suffisantes. Ce qui n'est pas et, à notre connaissance, n’a jamais été le cas à l'étranger. Une libéralisation tarifaire des études supérieures pousse les universités à privilégier les aides au mérite (pour bénéficier des effets de pairs) par rapport aux aides sociales et à entrer dans une logique cumulative susceptible d'accroître une double polarisation, d'une part entre des universités d'élite (attirant à la fois les meilleurs étudiants et les plus fortunés) et des collèges universitaires et, d'autre part, dans la représentation des classes sociales au sein de l'enseignement supérieur ${ }^{27}$. Parmi les perspectives de recherche qu'offre cet article, il apparaît donc fondamental de s'interroger, en matière d'équité, sur les conditions d'une péréquation entre universités ou sur la pertinence de grilles tarifaires nationales. Dans cette perspective, il apparaît également pertinent de s'interroger sur le caractère nécessaire- 
ment «synchrone " des contributions que l'on pourrait attendre des étudiants : ils pourraient en effet contribuer au financement de l'enseignement supérieur, non par des frais d'inscription pendant leur scolarité, mais par une taxe ou cotisation progressive, une fois en position d'activité.

Lorsque les tarifs sont laissés (relativement) libres de jouer le rôle de prix, nous montrons également que rien ne garantit un comportement efficient du marché. De nombreux travaux insistent sur le rôle des frais d'inscription pour inciter les différents acteurs à l'effort et à l'innovation ou pour sélectionner les étudiants et pour les orienter. Pourtant, la libéralisation ne tend pas naturellement vers l'efficience en présence de marchés imparfaits et d'une rationalité limitée des agents (information imparfaite, biais psycho-sociaux et capacité de traitement de cette information limitée) dont attestent des travaux empiriques. L'effet du poids de la dette, en lien avec le déterminisme social, distord les comportements individuels, non seulement dans le choix d'un cursus mais également dans celui des débouchés. Enfin, rien ne prouve qu'un marché de l'enseignement supérieur poussera ses différents acteurs à l'effort : les ressorts de la motivation sont en effet complexes, que ce soit pour les étudiants, les enseignantschercheurs ou les universités. Ils ne s'apparentent vraisemblablement pas à la simple maximisation d'une fonction de profit.

En matière de financement, nous soulignons que les frais d'inscription ne répondent pas plus au besoins des universités. L'Etat tend en effet généralement à se désengager à mesure que les frais d'inscription augmentent. De surcroît, les mécanismes d'accompagnement des frais d'inscription (généralement des prêts à remboursement contingent ou des bourses) peuvent se révéler coûteux pour les finances publiques.

Nous avons enfin souligné que les PARC, pour respecter le principe de compensation, doivent a minima permettre à l'étudiant de couvrir ses frais de scolarité (inscription, coût de la vie et coûts d'opportunité) afin qu'il ne soit pas obligé de travailler (source d'échec) et puisse aspirer aux filières auxquelles ses capacités lui permettent de prétendre, indépendamment de son milieu d'origine. Ce qui n'est en général pas le cas : le plafond 
d'emprunt peut se révéler trop bas, le poids de la dette distorsif et l'opportunisme accru du fait des mécanismes assurantiels qui leur sont associés. Parallèlement à cette financiarisation de l'enseignement supérieur, les aides sociales (déjà insuffisantes alors même que les exonérations fiscales sont anti-redistributives) auront tendance à se tarir. Il apparaît alors que, face à un choix éducatif, il n'est pas équivalent de bénéficier d'un financement familial et d'une possibilité d'emprunt. Aussi, face à la logique de capitalisation qui sous-tend la mise en ouvre de frais d'inscription, une piste de recherche pourrait consister en la théorisation de ce que pourrait être un régime d' "éducation par répartition" financé par la fiscalité nationale et fondé sur le financement récurrent des universités et une allocation universelle d'autonomie pour les étudiants. La définition d'un tel régime et de ses modalités de mise en œuvre dépasse néanmoins le cadre de cet article et constitue une perspective pour de futurs travaux.

Les auteurs tiennent à remercier les relecteurs anonymes pour leur travail et pour l'enrichissement de l'article rendu possible par leurs commentaires.

David Flacher est maître de conférences au CEPN - Université Paris 13 - Sorbonne Paris Cité et CNRS (UMR 7234).

Adresse : 99 avenue Jean-Bastiste Clément, 93430 Villetaneuse. Email : david@flacher.fr Hugo Harari-Kermadec est maître de conférences à l'IDHE - ENS Cachan et CNRS (UMR 8533).

Adresse : 61, avenue du Président Wilson, 94235 Cachan Cedex.

Léonard Moulin est doctorant au CEPN - Université Paris 13 - Sorbonne Paris Cité et CNRS (UMR 7234).

Adresse : 99 avenue Jean-Bastiste Clément, 93430 Villetaneuse. 


\section{Notes}

1. Voir, par exemple, le rapport du groupe de réflexion "Terra Nova ", proche de la gauche (Lichtenberger et Aïdara [2011]) ou les notes de l'Institut Montaigne, proche de la droite (Brandou [2011] et Colin [2008]).

2. Les bourses ne représentent, selon Eurostat [2007], que $8,2 \%$ des dépenses d'éducation. Les avantages fiscaux des plus aisés (notamment à travers le rattachement d'un enfant étudiant de plus de 21 ans) représentant $60 \%$ du montant des bourses dont profitent les ménages modestes (CERC [2003], Albouy et al. [2002], voir aussi la première partie de la deuxième section de cet article).

3. Calculs de Gregoir [2008] à partir des enquêtes Emploi de l'Insee. Des estimations sont également proposées par Courtioux ([2009], p. 7). A titre d'exemple, en 2005 , la dépense publique individuelle annuelle pour l'éducation supérieure allait de 4905 euros pour le Deug à 8486 euros pour la licence, 17805 euros pour un DEA et jusqu'à 127527 euros pour les " très grandes écoles " (génération de référence : 1970).

4. Il en résulte des taux de couverture individuels (définis comme les rapports entre les montants des retours fiscaux et des dépenses) très hétérogènes et essentiellement favorables aux formations longues et prestigieuses.

5. Fougère et al. [2009] montrent que l'occupation d'un emploi régulier pour un étudiant réduit significativement sa probabilité de réussir aux examens de fin d'année universitaire (cette diminution serait de l'ordre de 40\%).

6. Vinokur ([2009], p. 444) s'appuie sur les statistiques américaines du National
Center for Education Statistics montrant que les aides directes aux étudiants du quartile supérieur ont fortement augmenté entre 1995 et 1999 , alors que les aides aux étudiants issus de milieu à revenus plus modestes tendent à stagner.

7. L'hypothèse est contestable dans la mesure où l'investissement dans la préparation des examens ne constitue pas un investissement à perte s'il porte sur des savoirs essentiels ou réutilisables.

8. Ces deux approches sont en effet, en théorie, partiellement substituables. Fernàndez [1998] et Fernàndez et Gali [1999] proposent ainsi un modèle dans lequel il est possible d'allouer de manière efficiente les étudiants entre les universités d'élite et les universités standard, de manière décentralisée, en laissant le marché établir le prix des formations, ou de manière centralisée, à l'aide d'examens d'entrée. Néanmoins, le coût de préparation des examens, d'une part, et la contrainte d'endettement, d'autre part, constituent des limites respectivement à l'une et à l'autre des démarches.

9. Voir notamment Kahneman et al. [1982] ou Boudon [1994]. Des résultats similaires à ceux de Sullivan [2006] ont été mis en évidence par Caille et O’Prey ([2005], p. 50) : les étudiants évaluent leurs capacités avec un biais positif lorsqu'ils sont issus des classes sociales les plus favorisées et négatif lorsqu'ils proviennent d'une classe sociale moins favorisée. La norme sociale (au sein des classes les plus favorisées) peut induire des résistances à la tentation d'abandonner l'école après un échec (Gambetta [2009], p. 173).

10. Cf. Breen et Goldthorpe [1997] ou Holm et Jaeger [2008]. 
11. Pour Bourdieu [1974], « le rendement scolaire de l'action scolaire dépend du capital culturel préalablement investi par la famille et le rendement économique et social du titre scolaire dépend du capital social, lui aussi hérité, qui peut être mis à son service" (p.36).

12. Cette transformation des relations entre étudiants et universités en rapport marchand a été mise en évidence par Rotschild et White [1995], Maringe [2006], Nicolescu [2009], Voss et al. [2007]. "The introduction of or substantial increase in tuition fees in some countries have significant implications for student-university relations. Paying students conceived as customers rather than partners fits well into the emerging ideal of the modern corporate university " Brennan et Shah ([2011], p. 78).

13. Voir notamment Winston [1999] qui montre les limites d'une conception entrepreneuriale de l'université, alors que les coûts sont largement subventionnés, que les études constituent un bien d'expérience (donc risqué), que les bénéfices sont entièrement réinvestis dans l'institution ou l'aide aux étudiants et que les effets de pairs conduisent à rechercher le prestige plutôt que les profits. Voir aussi la note $\mathrm{n}^{\circ} 17$ sur les coûts de marketing.

14. Source : MEN-MESR-DEPP. Après 2006-2007, la LOLF intègre les dépenses des IUT aux budgets des universités

15. Source : Haaristo et al. ([2011], p. 1). En Lituanie, les frais d'inscription vont même jusqu'à peser $41 \%$ du budget total d'un étudiant.

16. D'après l'American Council of Education, la part du financement public dans les revenus des " colleges " publics est passée de $48 \%$ en 1980 à 35\% en 2000 (Vinokur ([2009], p. 445). De même, entre 2000 et 2008, les dépenses par étudiant ont diminué aux EtatsUnis (OCDE [2011], p. 231) alors que la participation financière des étudiants augmentait (OCDE [2011], p. 274). Au Royaume-Uni, les dépenses de l'Etat pour l'enseignement supérieur entre 2005 et 2008 ont diminué de plus de $18 \%$ (passant de 14,1 milliards de livres à 11,9 milliards de livres) pendant que les dépenses des ménages augmentaient, elles, de $54 \%$ (passant de 5,8 milliards de livres à 9,5 milliards de livres).

17. Aux Etats-Unis, le cabinet Lipman Hearne [2010] montre, à partir d'un échantillon de 212 établissements d'enseignement supérieur, que les dépenses de marketing ont augmenté de $99 \%$ à $208 \%$ en moyenne selon la taille de l'établissement entre 2001 et 2009. Durant cette même période les frais d'inscription ont augmenté de plus de $30 \%$ (NCES [2012]). Bien qu'il n'existe pas, à notre connaissance, de travaux qui établissent l'existence d'un lien direct entre l'augmentation des frais d'inscription et celle des dépenses de marketing, nous constatons que les deux évolutions sont liées et sommes amenés à penser qu'une partie de l'augmentation des recettes liée au relèvement des frais d'inscription permet d'accroître les dépenses de marketing des établissements. Des perspectives de recherche sur ce sujet sont ouvertes.

18. Nous laissons de côté les coûts d'opportunité dans la mesure où d'autres mécanismes d'aide existent ou pourraient être développés spécifiquement.

19. En France, le montant des bourses dépend du revenu annuel des parents et des points de charges de la famille (éloignement entre domicile familial et établissement, nombre d'enfants à charge, handicap...). Ces deux variables déterminent l'échelon auquel l'étudiant appartient. A l'exonération de droits universitaires et de cotisation à la sécurité sociale étudiante peuvent s'ajouter jusqu'à 4600 euros par année universitaire. Source : CNOUSCROUS et B.O n ${ }^{\circ} 13$ du 29 mars 2007. 
20. Un étudiant boursier percevra entre 1335 euros (échelon 1) et 3607 euros (échelon 5) lorsque les revenus de ses parents s'échelonnent entre 8490 euros et 16010 euros, tandis que les étudiants issus des milieux les plus favorisés bénéficieront de 1182 euros d'avantage fiscal (gain fiscal lié au rattachement d'un enfant étudiant) lorsque le revenu familial atteint 50000 euros annuel, et 2159 euros lorsque celui-ci atteint 100000 euros.

21. La part des coûts devant être remboursée peut varier en fonction des formations pour inciter les étudiants à s'orienter vers certaines filières. Ces variations peuvent reposer sur des différences dans les frais d'inscription ou sur les conditions d'accès au crédit.

22. Selon Courtioux et Gregoir [2010] parmi les déplômés du supérieur souscrivant à un PARC, seulement un sur mille pourrait être en situation d'aléa moral.

23. Le lecteur intéressé pourra se reporter à Vinokur ([2007], p. 221) sqq. et Vinokur ([2009], pp. 446-447), des références à l'appui de cette affirmation concernant les Etats-Unis. Voir Thompson et Bekhradnia [2010] pour l'Angleterre.

24. Sur le développement du marché financier autour des prêts étudiants, voir Vinokur [2007].
25. Pour Carneiro et Heckman [2002], la contrainte d'endettement ne concernerait réellement, à court terme, qu'au plus $8 \%$ de la population. Pour Frenette [2007], " les jeunes défavorisés décident souvent de ne pas fréquenter l'université parce qu'ils n'ont pas un bon rendement scolaire, ils ne désirent pas poursuivre leurs études, leurs amis sont moins susceptibles d'aller à l'université, etc. Même s'ils peuvent emprunter suffisamment d'argent, certains peuvent avoir une aversion pour les dettes $\mathrm{du}$ fait qu'ils sous-estiment les avantages économiques d'un diplôme universitaire sur toute une vie " (Frenette [2007], p. 9). Kane [1994] souligne aussi que l'inscription à l'université est particulièrement sensible aux frais d'inscription chez les étudiants appartenant aux classes sociales dont le revenu est le plus bas.

26. Voir aussi Vinokur [2007], pour qui " L'endettement des jeunes diplômés engendre des phénomènes migratoires cumulatifs, in situ en les détournant des emplois correspondant à leurs qualifications mais insuffisamment rémunérés (brain waste), et à l'étranger (brain drain)" (Vinokur [2007], p. 228). Le poids de la dette serait donc un déterminant de la fuite des cerveaux dans les migrations internationales (Vinokur [2006], Vinokur [2008]).

27. Voir en particulier les travaux de Epple et al. [2004] et de Hsieh et Urquiola [2006]. 


\section{Références}

P. Aghion et P. Howitt [2009] : The Economics of Growth, MIT Press, Cambridge.

V. Albouy, F. Bouton et N. Roth [2002] : Les transferts en faveur des familles : un bilan statique des transferts monétaires et des transferts éducatifs, séminaire CERC-DPDInsee, "Les effets redistributifs de l'éducation : les enseignements d'une approche monétaire statique ».

A. d'Autume, P. Le Merrer et J. PisaniFerry [2006] : Financement et organisation de l'enseignement supérieur : vers un nouveau modèle? Revue d'économie politique 116(6), pp. 743-744.

S. Barbaro [2005] : Equity and Efficiency Considerations of Public Higher Education, Lecture Notes in Economics and Mathematical Systems, Springer.

N. Barr [1993] : Alternative Funding Resources for Higher Education, The Economic Journal 103(418), pp. 718-728.

R. Bennett, H. Glennerster et D. Nevinson [1992] : Investing in Skill: To Stay on or not to Stay on ? Oxford Review of Economic Policy 8, pp. 130-145.

R. Boarini et H. Strauss [2007] : The Private Internal Rates of Return to Tertiary Education: New Estimates for 21 OECD Countries, working paper 591, OECD Economics Department.

R. Boudon [1974] : Education, Opportunity, and Social Inequality : Changing Prospects in Western Society, WileyInterscience, New York.

R. Boudon [1994] : The Art of Self-Persuasion : The Social Explanation of False Beliefs, Polity Press, Cambridge.
P. Bourdieu [1974] : Avenir de classe et causalité du probable, Revue française de sociologie 15 , pp. 3-42.

L.A. Bovenberg et B. Jacob [2005] : Redistribution and Education Subsidies Are Siamese Twins. Journal of Public Economics, 89(11-12), pp. 2005-2035.

M. Brandou [2011] : 2012 : Après l'autonomie, parlera-t-on du financement des universités ? Rapport technique, Institut Montaigne.

R. Breen et J.H. Goldthorpe [1997] : Explaining Educational Differentials : Towards a Formal Rational Action Theory, Rationality and Society 9(3), pp. 275-303.

J. Brennan and T. Shah [2011] : Higher Education and Society in Changing Times : Looking Back and Looking Forward, Center for Higher Education Research and Information.

T.O. Brodaty, R.J. Gary-Bobo et A. Prieto [2009] : Heterogeneity in Risk Aversion, Education and Wages, working paper TEAM, Université Paris 1.

J. Caille et S. O'prey [2005] : Estime de soi et réussite scolaire sept ans après l'entrée en sixième, Education \& formations 72 , pp. 25-52.

C. Callender [2006] : Access to Higher Education in Britain: The Impact of Tuition Fees and Financial Assistance, Higher Education Dynamics 14, pp. 105-132.

S.V. Cameron et J.J. Heckman [2001] : The Dynamics of Educational Attainment for Black, Hispanic, and White Males, Journal of Political Economy 109 (3), pp. 455-499.

D. Card [1999] : The Causal Effect of Education on Earnings, in O.C Ashenfelter 
et D. Card (eds.), Handbook of Labor Economics, Elsevier, vol. 3, chap. 30, pp. 1801-1863.

P. Carneiro et J.J. Heckman [2002] : The Evidence on Credit Constraints in Post-Secondary Schooling, The Economic Journal 112(482), pp. 705-734.

CERC [2003] : Education et redistribution, La documentation française, Paris.

N. Colin [2008] : Pour une contribution plus juste au système d'enseignement supérieur, rapport technique, Institut Montaigne.

Commission européenne [2006] : Faire réussir le projet de modernisation pour les universités : formation, recherche et innovation, communication de la Commission européenne 208.

P. Courtioux [2009] : Peut-on financer l'éducation du supérieur de manière plus équitable? working paper, EDHEC.

P. Courtioux [2010] : L'effet du système socio-fiscal sur les rendements privés de l'enseignement supérieur, working paper, EDHEC.

P. Courtioux et S. Gregoir [2010] : Le développement conjoint de droits d'inscription et de prêts conditionnels au revenu comme solution possible de financement de l'enseignement supérieur, séminaire Claude Fourgeaud, ministère des Finances et de l'industrie.

G. De Fraja [2002] : The Design of Optimal Education Policies, Review of Economic Studies 69, pp. 437-466.

M. Dynarski [1994] : Who Defaults on Student Loans? Findings from The National Postsecondary Student Aid Study, Economics of Education Review 13(1), pp. 55-68.

Z. Eckstein et K.I. Wolpin [1999] : Why Youths Drop Out of High School: The Impact of Preferences, Opportuni- ties, and Abilities, Econometrica 67(6), pp. 1295-1339.

D. Epple, D. Figlio et R. Romano [2004] : Competition Between Private and Public Schools : Testing Stratification and Pricing Predictions, Journal of Public Economics 88(7-8), pp. 1215-1245.

R. Fernàndez [1998] : Education and Borrowing Constraints : Tests vs. Prices, NBER working paper 6558.

R. Fernàndez et J. Gali [1999] : To Each According to... ? Tournaments, Markets and the Matching Problem Under Borrowing Constraints, Review of Economic Studies 66(4), pp. 799-824.

E. Field [2009] : Educational Debt Burden and Career Choice : Evidence from a Financial Aid Experiment at Nyu Law School, American Economic Journal : Applied Economics 1(1), pp. 1-21.

D. Flacher et H. Harari-Kermadec [2013] : Tuition Fees, Self-Esteem and Social Heterogeneity, Education Economics.

M. Fleurbaey [1995] : Equality and Responsibility. European Economic Review 39(3-4), pp. 683-689.

D. Fougère, M. Beffy et A. Maurel [2009] : Limpact du travail salarié des étudiants sur la réussite et la poursuite des études universitaires, Economie et statistique 422(1), pp. 31-50.

M. Frenette [2005] : L'incidence des frais de scolarité sur l'accès à l'université : résultats de la vaste déréglementation des frais de scolarité des programmes professionnels, Dir. des études analytiques : doc. de recherche 264, Statistique Canada.

M. Frenette [2007] : Pourquoi les jeunes provenant de familles à plus faible revenu sont-ils moins susceptibles de fréquenter l'université ? Analyse fondée sur les aptitudes aux études, linfluence des parents et les contraintes financières, Dir. des études 
analytiques : doc. de recherche 295, Statistique Canada.

M. Friedman [1962] : Capitalism and Freedom, IL : University of Chicago Press.

D. Gambetta [2009] : Were they Pushed or Did they Jump? Individual Decision Mechanisms in Education, Cambridge University Press.

E. Garces, D. Thomas et J. Currie [2002] : Longer-term Effects of Head Start, The American Economic Review 92(4), pp. 999-1012.

R.J. Gary-Bobo et A. Trannoy [2005] : Faut-il augmenter les droits d'inscription à l'université ? Revue française d'économie 19(3), pp. 189-237.

R.J. Gary-Bobo et A. Trannoy [2008] : Efficient Tuition Fees and Examinations, Journal of the European Economic Association 6(6), pp. 1211-1243.

B. Gazier [1992] : Economie du travail et de l'emploi, Dalloz (eds).

S. Gregoir [2008] : Les prêts étudiants peuvent-ils être un outil de progrès social ? working paper, EDHEC.

M. Grossman [2006] : Education and Nonmarket Outcomes, in E. Hanushek et F. Welch (eds.), Handbook of the Economics of Education, Elsevier, vol. 1, chap. 10, pp. 577-633.

M. Grossman et R. Kaestner [1997] : Effects of Education on Health, in J. Behrman et N. Stacey (eds.), The Social Benefits Of Education, University of Michigan Press, pp. 9-123.

H.S. Haaristo, D. Orr et B. Little [2011] : The Impact of Fees on Students'Budget, technical report, Eurostudent.

C. Harmon, H. Oosterbeek et I. Walker [2003] : The Returns to Education : Microeconomics, Journal of Economic Surveys 17(2), pp. 115-156.
R.H. Haveman et B.L. Wolfe [1984] : Schooling and Economic Well-Being : The Role of Nonmarket Effects, The Journal of Human Resources 19(3), pp. 377-407.

R. Haveman et B.L. Wolfe [1995] : The Determinants of Children's Attainments : A Review of Methods and Findings, Journal of Economic Literature 33(4), pp. 1829-1878.

J.C. Hearn [1991] : Academic and Nonacademic Influences on the College Destinations of 1980 High School Graduates, Sociology of Education 64(3), pp. 158-171.

L. Hendricks [2004] : A Database of Mincerian Earnings Regressions, www.lhendricks.org/Mincer.htm.

A. Holm et M.M. Jaeger [2008] : Does Relative Risk Aversion Explain Educational Inequality? A Dynamic Choice Approach, Research in Social Stratification and Mobility 26(3), pp. 199-219.

C.T. Hsieh et M. Urquiola [2006] : The Effects of Generalized School Choice on Achievement and Stratification : Evidence from Chile's Voucher Program, Journal of Public Economics 90(8-9), pp. 1477-1503.

G.E. Johnson [1984] : Subsidies for Higher Education, Journal of Labor Economics 2(3), pp. 303-318.

D.B. Johnstone [2004] : The Economics and Politics of Cost Sharing in Higher Education: Comparative Perspectives, Economics of Education Review 23(4), pp. 403-410.

D. Kahneman, P. Slovic et A. Tverski [1982] : Judgement Under Uncertainty : Heuristics and biases, Cambridge University Press.

T.J. Kane [1994] : College Entry by Blacks Since 1970: The Role of College Costs, Family Background, and the Returns to Education, The Journal of Political Economy 102(5), pp. 878-911. 
M.P. Keane et K.I. Wolpin [2001] : The Effect of Parental Transfers and Borrowing Constraints on Educational Attainment, International Economic Review 42(4), pp. 1051-1103.

A.B. Krueger et M. Lindahl [2001] : Education for Growth : Why and for Whom? Journal of Economic Literature 39(4), pp. 1101-1136.

Y. Lichtenberger et A. Aïdara [2011] : Faire réussir nos étudiants, faire progresser la France : propositions pour un sursaut vers la société de la connaissance, rapport technique 12, Terra Nova.

Lipman Hearne [2010] : Wondering What Works? The Changing Marketing Mix in Higher Education, A report on marketing spending at colleges and universities, www. lipmanhearne.com.

F. Maringe [2006] : University and Course Choice, International Journal of Educational Management, 20(6), pp. 466-479.

F. Marty [2004] : Déséquilibres et défaillances des marchés électriques : la libéralisation est-elle coupable?, L'Economie politique 24(4), pp. 43-58.

J. Micklewright [1989] : Choice at Sixteen, Economica 56(221), pp. 25-39.

NCES [2012] : Digest of Education Statistics, 2011, U.S. Department of Education, National Center for Education Statistics.

L. Nicolescu [2009] : Applying Marketing to Higher Education: Scope and Limits. Management \& Marketing 4(2), pp. 35-44.

OCDE [2009] : Regards sur l'éducation, OCDE publishing.

OCDE [2010] : Panorama des statistiques de l'OCDE, OCDE publishing.

OCDE [2011] : Regards sur l'éducation, OCDE publishing.
G. Orfield [1992] : Money, Equity, and College Access, Harvard Educational Review 62(3), pp. 337-373.

M. Palacios [2004] : Investing in Human Capital : A Capital Markets Approach to Student Funding, Cambridge University Press.

H. Pennell et A. West [2005] : The Impact of Increased Fees on Participation in Higher Education in England, Higher Education Quarterly 59(2), pp. 127-137.

E. Plug et W. Vijverberg [2005] : Does Family Income Matter for Schooling Outcomes ? Using Adoption as a Natural Experiment, Economic Journal 115(506), pp. 879-906.

J. Rawls [1971] : A Theory of Justice, Harvard University Press, Cambridge (MA).

M. Rotschild and L.J. White [1995] : The Analytics of Pricing Higher Education and Other Servics in with the Customers Are Inputs, Journal of Political Economy 103(3), pp. 573-586.

A. Sen [2010] : L'idée de justice, Flammarion, Paris.

M. Spence [1973] : Job Market Signaling, The Quarterly Journal of Economics, 87 (3), pp. 355-374.

J.E. Stiglitz [1975] : The Theory of " Screening " Education, and the Distribution of Income, The American Economic Review 65(3), pp. 283-300.

H. Strauss et C. de La Maisonneuve [2009] : The Wage Premium on Tertiary Education: New Estimates for 21 OECD Countries, OECD Journal : Economic Studies 2009(1), pp. 183-210.

A. Sullivan [2006] : Students as Rational Decision-Makers : The Question of Beliefs and Attitudes, London Review of Education 4(3), pp. 271-290. 
J.R.W. Temple [2001] : Effets de l'éducation et du capital social sur la croissance dans les pays de l'OCDE, Revue économique de l'OCDE 2001(2), pp. 59-110.

J. Thompson et B. Bekhradnia [2010] : The Government's Proposals for Higher Education Funding and Student Finance, technical report, Higher Education Policy Institute.

A. Trannoy [2006] : Financement des universités, financement des études, Revue d'économie politique 116(6), pp. 745-782.

V. Vandenberghe et O. Debande [2008] : Refinancing Europe's Higher Education Through Deferred and Income-Contingent Fees : An Empirical Assessment Using Belgian, German \& UK Data, European Journal of Political Economy 24, pp. 364-386.

A. Vinokur [2006] : Brain Migration Revisited, Globalisation, Societies and Education 4(1), pp. 7-24.
A. Vinokur [2007] : Study Now, Pay Later : partage des coûts, endettement étudiant et restructuration de l'enseignement supérieur, in Annie Vinokur (eds.), Pouvoirs et financement en éducation, L'Harmattan, Paris.

A. Vinokur [2008] : De la mobilité des cerveaux, Revue formation-emploi 103 , pp. 9-21.

A. Vinokur [2009] : La querelle des droits d'inscription, Revue du MAUSS 33(1), pp. 441-453.

R. Voss, T. Gruber et I. Szmigin [2007] : Service Quality in Higher Education : The Role of Student Expectations, Journal of Business Research 60(9), pp. 949-959.

G.C. Winston [1999] : Subsidies, Hierarchy and Peers: The Awkward Economics of Higher Education, The Journal of Economic Perspective 13(1), pp. 13-36. 